\title{
Dimension (In)equalities and Hölder Continuous Curves in Fractal Percolation
}

\author{
Erik I. Broman • Federico Camia • \\ Matthijs Joosten • Ronald Meester
}

Received: 16 May 2011 / Revised: 14 February 2012 / Published online: 23 March 2012

(C) The Author(s) 2012. This article is published with open access at Springerlink.com

\begin{abstract}
We relate various concepts of fractal dimension of the limiting set $\mathcal{C}$ in fractal percolation to the dimensions of the set consisting of connected components larger than one point and its complement in $\mathcal{C}$ (the "dust"). In two dimensions, we also show that the set consisting of connected components larger than one point is almost surely the union of non-trivial Hölder continuous curves, all with the same exponent. Finally, we give a short proof of the fact that in two dimensions, any curve in the limiting set must have Hausdorff dimension strictly larger than 1 .
\end{abstract}

Keywords Fractal percolation - Hausdorff dimension - Box counting dimension · Hölder continuous curves $\cdot$ Subsequential weak limits

Mathematics Subject Classification $60 \mathrm{~K} 35 \cdot 28 \mathrm{~A} 80 \cdot 37 \mathrm{~F} 35 \cdot 54 \mathrm{C} 05$

\section{Introduction and Main Results}

In this paper we are concerned with a percolation model, first introduced in [12], which is known as Mandelbrot's fractal percolation process and which can be informally described as follows. For any integers $d \geq 2$ and $N \geq 2$, we start by dividing the

\footnotetext{
E.I. Broman

Uppsala Universitet, Uppsala, Sweden

e-mail: broman@math.uu.se

F. Camia $\cdot$ M. Joosten $(\varangle) \cdot$ R. Meester

VU University Amsterdam, Amsterdam, The Netherlands

e-mail: matthijsjoosten@gmail.com

F. Camia

e-mail: f.camia@vu.nl

R. Meester

e-mail: r.w.j.meester@vu.nl 
unit cube $[0,1]^{d} \subset \mathbb{R}^{d}$ into $N^{d}$ closed subcubes of equal size $1 / N \times 1 / N \times \cdots \times 1 / N$. Given $p \in[0,1]$ and a subcube, we retain the subcube with probability $p$ and discard it with probability $1-p$. This is done independently for every subcube of the partition. Sometimes we adopt the terminology calling retained cubes black and deleted cubes white. We define the random set $\mathcal{C}_{N}^{1}=\mathcal{C}_{N}^{1}(d, p) \subset[0,1]^{d}$ as the union of all retained subcubes. Next consider any retained (assuming that $\mathcal{C}_{N}^{1} \neq \emptyset$ ) subcube $B$ in $\mathcal{C}_{N}^{1}$. We repeat the described procedure on a smaller scale by dividing $B$ into $N^{d}$ further subcubes, discarding or retaining them as above. We do this for every retained subcube of $\mathcal{C}_{N}^{1}$. This yields a new random set $\mathcal{C}_{N}^{2} \subset \mathcal{C}_{N}^{1}$. Iterating the procedure on every smaller scale yields an infinite sequence of random sets $[0,1]^{d} \supset \mathcal{C}_{N}^{1} \supset \mathcal{C}_{N}^{2} \supset \cdots$ and we define the limiting set

$$
\mathcal{C}_{N}:=\bigcap_{n=1}^{\infty} \mathcal{C}_{N}^{n} .
$$

We will hereafter suppress the $N$ in our notation and simply write $\mathcal{C}$ for $\mathcal{C}_{N}$.

We will need a more formal definition of the model as well. Let

$$
I_{k}^{n}:=\left[\frac{(k-1)}{N^{n}}, \frac{k}{N^{n}}\right],
$$

where $n \geq 1$ and $1 \leq k \leq N^{n}$. For $\mathbf{k}=\left(k_{1}, \ldots, k_{d}\right)$, consider the subcube $D_{\mathbf{k}}^{n}$ of $[0,1]^{d}$ defined by $D_{\mathbf{k}}^{n}:=I_{k_{1}}^{n} \times I_{k_{2}}^{n} \times \cdots \times I_{k_{d}}^{n}$, and let $D:=\left\{D_{\mathbf{k}}^{n}: n \geq 1,1 \leq k_{l} \leq N^{n}\right\}$. A cube $D_{\mathbf{k}}^{n}$ will sometimes be called a level- $n$ cube. We define the sample space by

$$
\Omega:=\{0,1\}^{D},
$$

and denote an element of $\Omega$ by $\omega$. We let $\mathcal{B}$ be the Borel $\sigma$-algebra on $\Omega$ generated by the cylinders and let $\mathbb{P}_{p}$ denote the product measure on $\mathcal{B}$ with density $p \in[0,1]$, that is, we let $\mathbb{P}_{p}\left(\omega\left(D_{\mathbf{k}}^{n}\right)=1\right)=p$ independently for every $D_{\mathbf{k}}^{n} \in D$. The limiting set is then defined to be the intersection of all $D_{\mathbf{k}}^{n} \in D$ such that $\omega\left(D_{\mathbf{k}}^{n}\right)=1$.

Let $C R\left([0,1]^{d}\right)$ denote the event that $\mathcal{C}$ contains a connected component which intersects the left-hand side $\{0\} \times[0,1]^{d-1}$ of the unit cube and also intersects the right-hand side $\{1\} \times[0,1]^{d-1}$. In this case we say that a left-right crossing of the unit cube occurs.

We define the percolation function $\theta_{N, d}$ by

$$
\theta_{N, d}(p):=\mathbb{P}_{p}\left(C R\left([0,1]^{d}\right)\right) .
$$

The critical value is defined as

$$
\tilde{p}_{c}=\tilde{p}_{c}(N, d):=\inf \left\{p: \theta_{N, d}(p)>0\right\} .
$$

It has been shown in [8] that the phase transition in Mandelbrot's fractal percolation is non-trivial, i.e. $0<\tilde{p}_{c}(N, d)<1$. Furthermore, it was discovered in [8] that for $d=2, \theta_{N, d}(p)$ is discontinuous at $\tilde{p}_{c}$ (see [10] for an easy proof). This was generalized in [4] to all $d \geq 3$ and $N$ large enough but the result is conjectured to hold for all $N$ in any dimension. (At this point we remark that as a corollary to the proof of 
Theorem 1.1 below, we obtain an explicit bound for the size of the discontinuity at the critical value $p_{c}$, defined below and conjectured to coincide with $\tilde{p}_{c}$, in terms of the Hausdorff dimension of the set $\mathcal{C}^{c}$, also to be defined below.)

In $d=2$, the set $\mathcal{C}$ is a.s. totally disconnected for $p<\tilde{p}_{c}(N, 2)$. This is also known to be true in higher dimensions for the same set of $N$ for which it is known that $\theta_{N, d}(p)$ is discontinuous at $\tilde{p}_{c}$ (see [5]) and is conjectured to be true for all $d$ and $N$. It is therefore natural to work with the following critical value:

$$
p_{c}(N, d):=\sup \{p: \mathcal{C} \text { is a.s. totally disconnected }\} .
$$

It is known (see [5]) that for any $d \geq 2$ and $N \geq 2$,

$$
\mathbb{P}_{p}(\mathcal{C} \text { is not totally disconnected })>0
$$

if $p=p_{c}(N, d)$. Given this, it is an easy exercise to show that for $p \geq p_{c}(N, d)$, $\mathcal{C} \neq \varnothing$ implies that the set $\mathcal{C}^{c}$ consisting of the union of all connected components larger than one point is a.s. not empty.

We now fix $N, d \geq 2$ and assume that $p_{c}(N, d) \leq p<1$. For any point $x \in \mathcal{C}$, let $\mathcal{C}_{x} \subset \mathcal{C}$ be the set of points $y \in \mathcal{C}$ that are connected to $x$ in $\mathcal{C}$. We call $\mathcal{C}_{x}$ the connected component of $x$. It is known (see [13]) that for $p \geq p_{c}(N, d)$ there exist a.s. uncountably many $x \in \mathcal{C}$ such that $\mathcal{C}_{x}=\{x\}$. We partition $\mathcal{C}$ into two sets, $\mathcal{C}^{d}:=$ $\left\{x \in \mathcal{C}: \mathcal{C}_{x}=\{x\}\right\}$ and the aforementioned $\mathcal{C}^{c}:=\mathcal{C} \backslash \mathcal{C}^{d}$. (To understand the notation: $d$ is short for "dust", and $c$ is short for "connected".)

Before we can state our results we will need some more definitions. The reader is referred to [11] for a general overview of the subject of fractal sets.

A countable collection $\left\{B_{i}\right\}_{i=1}^{\infty}$ of subsets of $\mathbb{R}^{d}$ with diameter at most $\epsilon$ is called an $\epsilon$-cover of $F$ if $F \subset \bigcup_{i=1}^{\infty} B_{i}$. Define the $s$-dimensional Hausdorff measure of $F$ as follows:

$$
\mathcal{H}^{s}(F):=\lim _{\epsilon \rightarrow 0} \inf \left\{\sum_{i=1}^{\infty} \operatorname{diam}\left(B_{i}\right)^{s}:\left\{B_{i}\right\}_{i=1}^{\infty} \text { is an } \epsilon \text {-cover of } F\right\} .
$$

The Hausdorff dimension $\operatorname{dim}_{\mathcal{H}}(F)$ of $F$ is defined as

$$
\operatorname{dim}_{\mathcal{H}}(F):=\inf \left\{s: \mathcal{H}^{s}(F)=0\right\},
$$

which also turns out to be equal to $\sup \left\{s: \mathcal{H}^{s}(F)=\infty\right\}$. The Hausdorff dimension of the limiting set in fractal percolation is a.s. given by the following equation, whose proof can be found in [8] or [11], Proposition 15.4:

$$
\operatorname{dim}_{\mathcal{H}}(\mathcal{C})= \begin{cases}d+\frac{\log p}{\log N} & \text { if } \mathcal{C} \neq \emptyset \\ 0 & \text { otherwise }\end{cases}
$$

There are many other concepts of dimensionality and we will in particular use the following. For a bounded set $F \subset \mathbb{R}^{d}$ let $M_{\delta}(F)$ be the minimal number of closed cubes of side length $\delta$ that is needed to cover $F$. 
The Lower Box counting dimension of $F \subset \mathbb{R}^{d}$ is given by

$$
\underline{\operatorname{dim}}_{B}(F):=\liminf _{\delta \rightarrow 0} \frac{\log M_{\delta}(F)}{-\log \delta},
$$

while the Upper Box counting dimension of $F \subset \mathbb{R}^{d}$ is given by

$$
\overline{\operatorname{dim}}_{B}(F):=\limsup _{\delta \rightarrow 0} \frac{\log M_{\delta}(F)}{-\log \delta} .
$$

If $\underline{\operatorname{dim}}_{B}(F)=\overline{\operatorname{dim}}_{B}(F)$ then the common value is denoted $\operatorname{dim}_{B}(F)$ and called the Box counting dimension of $F$. It is known (see e.g. [11]) that for any bounded set $F \subset \mathbb{R}^{d}$

$$
\operatorname{dim}_{\mathcal{H}}(F) \leq \underline{\operatorname{dim}}_{B}(F) \leq \overline{\operatorname{dim}}_{B}(F) .
$$

The next two theorems contain our dimension results for fractal percolation.

Theorem 1.1 For $p_{c}(N, d) \leq p<1$, we a.s. have

$$
\operatorname{dim}_{B}\left(\mathcal{C}^{c}\right)=\operatorname{dim}_{B}(\mathcal{C})=\operatorname{dim}_{\mathcal{H}}(\mathcal{C})
$$

If $\mathcal{C} \neq \varnothing$ then a.s.

$$
\operatorname{dim}_{\mathcal{H}}\left(\mathcal{C}^{c}\right)<\operatorname{dim}_{\mathcal{H}}(\mathcal{C}),
$$

from which it easily follows that a.s.

$$
\operatorname{dim}_{\mathcal{H}}\left(\mathcal{C}^{d}\right)=\operatorname{dim}_{\mathcal{H}}(\mathcal{C})
$$

Note that if $p<p_{c}(N, d), \mathcal{C}^{c}=\emptyset$ a.s. and so (6) still holds as long as $\operatorname{dim}_{\mathcal{H}}(\mathcal{C})>0$.

Theorem 1.2 For every $p$ there exists $1 \leq \beta=\beta(p) \leq d$ such that

$$
\mathbb{P}_{p}\left(\mathcal{C}^{c}=\emptyset \text { or } \operatorname{dim}_{\mathcal{H}}\left(\mathcal{C}^{c}\right)=\beta\right)=1
$$

For $\epsilon>0$, let $\mathcal{C}^{c, \epsilon}$ be the union of the connected components of diameter at least $\epsilon$. The following result suggests that the "small components" of $\mathcal{C}^{c}$ are the ones which actually determine its Box counting dimension.

Proposition 1.3 If $\mathcal{C}^{c} \neq \emptyset$, then

$$
\mathbb{E}_{p}\left[\underline{\operatorname{dim}}_{B}\left(\mathcal{C}^{c, \epsilon}\right)\right] \leq D \operatorname{dim}_{B}\left(\mathcal{C}^{c}\right)
$$

where $D<1$ is independent of $\epsilon$.

When $p \geq \tilde{p}_{c}$, it is natural to ask about the nature of the left-right crossings of the unit cube. For $d=2$, it was shown in [13] that $\mathcal{C}$ contains at least one continuous curve crossing the square as soon as a connected component crossing the square 
Fig. 1 The interface curves are drawn with broken lines. Arrows indicate the orientation

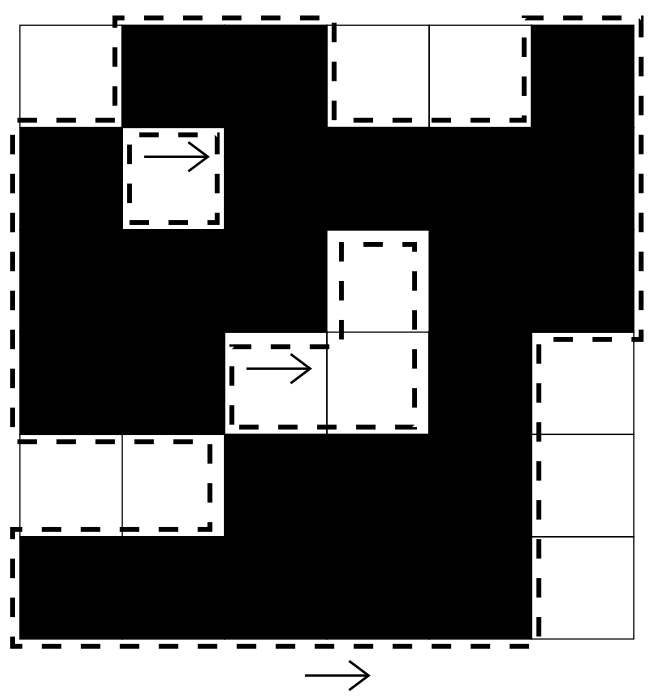

exists. It was later established in [7] (again for $d=2$ ) that any curve in $\mathcal{C}$ must have Hausdorff dimension strictly larger than 1.

In this paper, focusing again on the two-dimensional version of the model, we take the issue of the existence of continuous curves in $\mathcal{C}$ much further, using the sophisticated machinery of Aizenman and Burchard [1]. Their paper deals with scaling limits of systems of random curves, but we will show how their results can be useful in the context of fractal percolation as well. This is perhaps somewhat surprising, since the scaling limits in [1] deal with convergence in distribution, whereas in the fractal context, the fractal limiting set is an a.s. limit. The key will be a very careful comparison between convergence in the weak sense of curves in an appropriate topology, and convergence in the a.s. sense of compact sets in another topology. From such a comparison, one can obtain information about the compact sets that make up the a.s. limit of the fractal construction.

In order to state our results, we need some definitions. First of all, we define interface curves in the fractal process. The complement $\mathbb{R}^{2} \backslash \mathcal{C}^{n}$ consists of a finite number of connected components, exactly one of which is unbounded. The boundary of any such connected component can be split into closed curves (loops). We call such loops interface curves and denote by $\mathcal{F}_{n}$ the collection of interface curves after $n$ iterations of the fractal process. In order for our interface curves to be uniquely defined, we orient them in such a way that they have black (retained) squares on the left and white (discarded) squares on the right, and assume that they turn to the right at corners where two white and two black squares meet in a checkerboard configuration, see Fig. 1 .

A connected subset of an interface curve delimited by a starting and an ending point will be called an interface segment.

We continue with some general definitions concerning curves, mostly taken from [1] (see also [6]). We regard curves in $[0,1]^{2}$ as equivalence classes of continuous functions from $[0,1]$ to $[0,1]^{2}$ modulo strictly monotonic re-parameterizations. Be- 
low, $\gamma$ will represent a particular curve and $\gamma(t)$ a particular parameterization of $\gamma$. Denote by $\mathcal{S}$ the complete separable metric space of curves in $[0,1]^{2}$ with metric

$$
\mathrm{D}\left(\gamma_{1}, \gamma_{2}\right):=\inf \sup _{t \in[0,1]}\left|\gamma_{1}(t)-\gamma_{2}(t)\right|
$$

where the infimum is over all parameterizations of $\gamma_{1}$ and $\gamma_{2}$. The distance between two sets $\mathcal{F}$ and $\mathcal{F}^{\prime}$ of curves is defined by the Hausdorff metric induced by $\mathrm{D}$, that is, $\operatorname{Dist}\left(\mathcal{F}, \mathcal{F}^{\prime}\right) \leq \varepsilon$ if and only if

$$
\forall \gamma \in \mathcal{F}, \exists \gamma^{\prime} \in \mathcal{F}^{\prime} \quad \text { with } \mathrm{D}\left(\gamma, \gamma^{\prime}\right) \leq \varepsilon \text { and vice versa. }
$$

The space $\Sigma$ of closed subsets of $\mathcal{S}$ with the metric Dist is also a complete separable metric space.

The fractal process induces a probability measure $\mu_{n}$ on $\Sigma$, where $\mu_{n}$ denotes the distribution of $\mathcal{F}_{n}$. With this notation, we can present our main result on continuous curves in $\mathcal{C}$.

Theorem 1.4 The sequence of measures $\left(\mu_{n}\right)$ has subsequential weak limits. Any such weak limit $\mu$ assigns probability 1 to curve configurations in which all curves are Hölder continuous with the same exponent. The limiting set $\mathcal{C}$ has the same distribution as $g(\mathcal{F}):=\bigcup_{\gamma \in \mathcal{F}} \operatorname{Image}(\gamma)$, where $\mathcal{F}$ is a random set of curves distributed as $\mu$. In other words, $\mathcal{C}$ is distributed as the union of the images of the curves in a sample from a weak limit of the $\left(\mu_{n}\right)$.

Remark Note that, although Theorem 1.4 only claims the existence of subsequential weak limits for $\mu_{n}$ as $n \rightarrow \infty$, Theorem 1.4 combined with the existence of a unique a.s. limit for $\mathcal{C}^{n}$ implies that $g \circ \mu_{n}(\cdot)=\mu_{n}\left(g^{-1}(\cdot)\right)$ has a unique weak limit.

We now briefly discuss this result. Since a single point is of course a Hölder continuous curve, the bare statement that a set is the union of Hölder continuous curves is in itself close to being an empty statement. However, the curves in $\mathcal{C}$ mentioned in Theorem 1.4 cannot be exclusively curves whose image is one point. One way to see this is to rephrase the notion of weak convergence as follows (see also [1]). A sequence of probability measures $\left(\mu_{n}\right)$ on $\Sigma$ converges weakly to a probability $\mu$ measure on $\Sigma$ if and only if there exists a family of probability measures $\rho_{n}$ on $\Sigma \times \Sigma$ such that the first marginal of $\rho_{n}$ is $\mu_{n}$, the second marginal of $\rho_{n}$ is $\mu$ (for all $n$ ), and

$$
\int_{\Sigma \times \Sigma} \operatorname{Dist}\left(\mathcal{F}_{n}, \mathcal{F}\right) d \rho_{n}\left(\mathcal{F}_{n}, \mathcal{F}\right) \rightarrow 0
$$

as $n \rightarrow \infty$. It is now also clear what happens to the points in the "dust set" $\mathcal{C}^{d}:$ these are accounted for as well in the theorem, since any point $x \in \mathcal{C}^{d}$ can be approximated by curves in $\mathcal{F}_{n}$ whose diameter and distance to $x$ converge to 0 .

It is also possible to specialize to certain particular curves. As an example, we discuss the lowest crossing in $\mathcal{C}$ which we will first properly define. Condition on the existence in $\mathcal{C}^{n}$ of a left-right crossing of the unit square for all $n$, and consider 
the lowest interface segment $\sigma_{n}$ in $\mathcal{F}_{n}$ connecting the left and right sides of the unit square. The closure of the region in the unit square above $\sigma_{n}$ is a compact set (in the Euclidean topology) which decreases in $n$ and which therefore converges as $n \rightarrow \infty$. The lowest crossing in $\mathcal{C}$ is defined as the boundary of this limiting set.

Theorem 1.5 If $\mathcal{C}$ contains a left-right crossing of the unit square, then the lowest crossing in $\mathcal{C}$ is a Hölder continuous curve.

The machinery of Aizenman and Burchard also allows for a quick proof, given in Sect. 4, of the following result, first proved in [7].

Theorem 1.6 In two dimensions, there exists a constant $\kappa>1$ such that all continuous curves in $\mathcal{C}^{c}$ have Hausdorff dimension at least $\kappa$.

\section{Proofs of Theorems 1.1, 1.2 and Proposition 1.3}

Proof of Theorem 1.1 We start with the second equality of (5). Let $Z_{n}$ be the number of cubes of side length $N^{-n}$ that are retained in $\mathcal{C}^{n}$. First observe that

$$
\frac{\log Z_{n}}{-\log N^{-n}}=\frac{\log Z_{n}^{1 / n}}{\log N} .
$$

It is well known from the theory of branching processes (see e.g. [2, 9]) that $Z_{n}^{1 / n} \rightarrow$ $p N^{d}$ a.s. on the event $\mathcal{C} \neq \emptyset$. Therefore, for a.e. $\omega$ such that $\mathcal{C}(\omega) \neq \emptyset$,

$$
\lim _{n \rightarrow \infty} \frac{\log Z_{n}}{-\log N^{-n}}=d+\frac{\log p}{\log N},
$$

and hence $\overline{\operatorname{dim}}_{B}(\mathcal{C}) \leq d+\frac{\log p}{\log N}$. Equations (3) and (4) then imply that $\operatorname{dim}_{B}(\mathcal{C})=$ $\operatorname{dim}_{\mathcal{H}}(\mathcal{C})$.

For the first equality of (5), let $\left\{B_{i}\right\}_{i=1}^{M_{\delta}}$ be a cover of $\mathcal{C}^{c}$ using the minimal number $M_{\delta}$ of closed cubes of side length $\delta$. For $A, B \subset \mathbb{R}^{d}$, define $d(A, B):=$ $\inf \{|x-y|: x \in A, y \in B\}$, with $|\cdot|$ denoting Euclidean distance. Assume that there exists $x \in \mathcal{C}$ such that $d\left(x, \bigcup_{i=1}^{M_{\delta}} B_{i}\right)>0$. Then there must exist some $D_{\mathbf{k}}^{n}$ such that $d\left(D_{\mathbf{k}}^{n}, \bigcup_{i=1}^{M_{\delta}} B_{i}\right)>0$ and $x \in D_{\mathbf{k}}^{n}$, which implies that $\omega\left(D_{\mathbf{k}}^{n}\right)=1$ (i.e., $D_{\mathbf{k}}^{n}$ is retained). However, because of the scale-invariant construction of $\mathcal{C}$ and the fact that, for $p \geq p_{c}(N, d)$, a.s. $\mathcal{C}$ is either empty or contains connected components larger than one point (see [5]), $\mathcal{C} \cap D_{\mathbf{k}}^{n}$ must contain connected components larger than one point. This contradicts the fact that $\left\{B_{i}\right\}_{i=1}^{M_{\delta}}$ is a cover of $\mathcal{C}^{c}$ and shows that such an $x$ cannot exist. Furthermore, since the union $\bigcup_{i=1}^{M_{\delta}} B_{i}$ is closed, it follows that, if $d\left(x, \bigcup_{i=1}^{M_{\delta}} B_{i}\right)=0$ for $x \in \mathcal{C}$, then $x \in \bigcup_{i=1}^{M_{\delta}} B_{i}$. Therefore, $M_{\delta}$ must be the minimal number of closed cubes of side length $\delta$ that covers $\mathcal{C}$. This concludes the proof of (5). 
Since $\mathcal{C}=\mathcal{C}^{c} \cup \mathcal{C}^{d},(7)$ follows from (6) and the fact that (see [11])

$$
\operatorname{dim}_{\mathcal{H}}(\mathcal{C})=\max \left(\operatorname{dim}_{\mathcal{H}}\left(\mathcal{C}^{c}\right), \operatorname{dim}_{\mathcal{H}}\left(\mathcal{C}^{d}\right)\right)
$$

We proceed therefore by proving (6), inspired by an argument suggested by Lincoln Chayes to the second author. Recall that $\mathcal{C}^{c, \epsilon}$ is the union of the connected components of diameter at least $\epsilon$. For $p \geq p_{c}(N, d)$, we have that

$$
\operatorname{dim}_{\mathcal{H}}\left(\mathcal{C}^{c}\right)=\sup _{\epsilon} \operatorname{dim}_{\mathcal{H}}\left(\mathcal{C}^{c, \epsilon}\right)
$$

which is an easy consequence of the definition of Hausdorff dimension (see [11]). Therefore, it suffices to find an upper bound of $\operatorname{dim}_{\mathcal{H}}\left(\mathcal{C}^{c, \epsilon}\right)$ which is uniform in $\epsilon$ and strictly smaller than $\operatorname{dim}_{\mathcal{H}}(\mathcal{C})$.

We will now assume that $N \geq 5$ is odd. This assumption will make certain definitions easier to write down: the reader can check that the squares $D_{\mathbf{k}}^{n}$ below would not be uniquely defined for even $N$. We leave it to the reader to adapt the proof for all cases $N \geq 2$.

For $D_{\mathbf{k}}^{1}$ such that $(1 / 2, \ldots, 1 / 2) \in D_{\mathbf{k}}^{1}$, let $B\left(D_{\mathbf{k}}^{1} ; 1\right):=[0,1]^{d}$ and $B\left(D_{\mathbf{k}}^{1} ; 3 N^{-1}\right)$ be the two cubes concentric to $D_{\mathbf{k}}^{1}$ with side lengths 1 and $3 N^{-1}$, respectively. Let $\varphi_{N, d}(p)$ be the probability that there exists a connected component of $\mathcal{C}$ that crosses the "shell" $B\left(D_{\mathbf{k}}^{1} ; 1\right) \backslash B\left(D_{\mathbf{k}}^{1} ; 3 N^{-1}\right)$. It follows from [5] that $\varphi_{N, d}(p)>0$ whenever $p \geq p_{c}(N, d)$.

Assuming that $\mathcal{C}^{c} \neq \varnothing$, fix $\epsilon>0$ such that $\mathcal{C}^{c}$ contains at least one component of diameter larger than $\epsilon$ and let $l$ be such that $N^{-l+1} \leq \epsilon / d$. Consider a cube $D_{\mathbf{k}}^{n}$ for $n \geq l$ which is intersected by a component of $\mathcal{C}^{c}$ of diameter larger than $\epsilon$. Let $B\left(D_{\mathbf{k}}^{n} ; 3 N^{-n}\right)$ and $B\left(D_{\mathbf{k}}^{n} ; N^{-n+1}\right)$ be two cubes which are concentric to $D_{\mathbf{k}}^{n}$ and have side lengths $3 N^{-n}$ and $N^{-n+1}$, respectively. Obviously, for $D_{\mathbf{k}}^{n}$ to be intersected by a connected component of diameter larger than $\epsilon$, there must be a crossing of the shell $B\left(D_{\mathbf{k}}^{n} ; N^{-n+1}\right) \backslash B\left(D_{\mathbf{k}}^{n} ; 3 N^{-n}\right)$. (Note that, depending on the position of $D_{\mathbf{k}}^{n}$, it is possible that $B\left(D_{\mathbf{k}}^{n} ; 3 N^{-n}\right)$ and/or $B\left(D_{\mathbf{k}}^{n} ; N^{-n+1}\right)$ are only partially contained in $[0,1]^{d}$.)

We will now construct a specific cover of $\mathcal{C}^{c, \epsilon}$ which we will use in our estimate for its Hausdorff dimension. Let $W_{n}$ denote the set of cubes $D_{\mathbf{k}}^{n}$ with the following two properties:

- The intersection of all retained cubes of level $n$ and higher contains a crossing of the shell $B\left(D_{\mathbf{k}}^{n} ; N^{-n+1}\right) \backslash B\left(D_{\mathbf{k}}^{n} ; 3 N^{-n}\right)$. In other words, if we would make all cubes black until level $n-1$ (inclusive), then there would be a connected component in $\mathcal{C}$ crossing the shell $B\left(D_{\mathbf{k}}^{n} ; N^{-n+1}\right) \backslash B\left(D_{\mathbf{k}}^{n} ; 3 N^{-n}\right)$.

- $D_{\mathbf{k}}^{n}$ is retained, that is, $\omega\left(D_{\mathbf{k}}^{n}\right)=1$.

By scale invariance and independence between the two conditions, we have that $\mathbb{P}_{p}\left(D_{\mathbf{k}}^{n} \in W_{n}\right) \leq p \varphi_{N, d}(p)$. The inequality is due to a boundary effect since, as mentioned earlier, $B\left(D_{\mathbf{k}}^{n} ; 3 N^{-n}\right)$ and/or $B\left(D_{\mathbf{k}}^{n} ; N^{-n+1}\right)$ need not be completely contained in $[0,1]^{d}$.

For a given cube $D_{\mathbf{k}}^{n}$, let $B^{m}$ denote the level- $m$ cube which contains $D_{\mathbf{k}}^{n}$, where $m \leq n$ (with $B^{n}=D_{\mathbf{k}}^{n}$ ). We make two observations: 
Fig. 2 The cube $D_{k}^{n}$ is in $V_{n}$ and $D_{k}^{n+1}$, drawn with broken lines, belongs to $V_{n+1}$. Note that the corresponding shells are disjoint

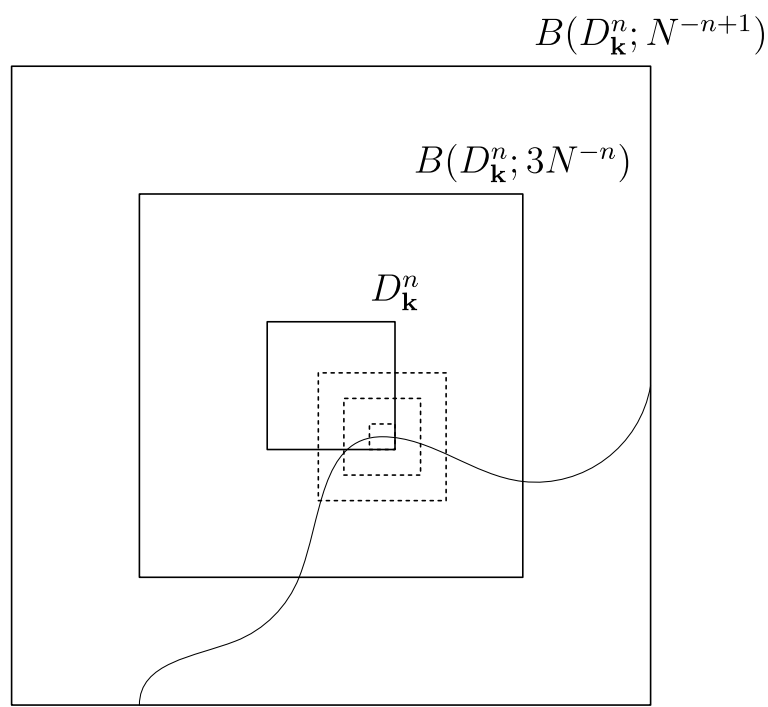

1. If $D_{\mathbf{k}}^{n}$ has a non-empty intersection with $\mathcal{C}^{c, \epsilon}$, then we have that $B^{m} \in W_{m}$, for all $m=l, l+1, \ldots, n$.

2. The events $\left\{B^{m} \in W_{m}\right\}$ form a collection of independent events; see Fig. 2.

This motivates us to define $V_{n}$ as the collection of cubes $D_{\mathbf{k}}^{n}$ for which the corresponding cubes $B^{m}$ are in $W_{m}$, for all $m=l, l+1, \ldots, n$. From observation 1 , we have that the collection $V_{n}$ forms a cover of $\mathcal{C}^{c, \epsilon}$. We can now write, using observation 2 ,

$$
\begin{aligned}
\mathbb{P}_{p}\left(D_{\mathbf{k}}^{n} \in V_{n}\right) & =\mathbb{P}_{p}\left(\bigcap_{m=l}^{n} B^{m} \in W_{m}\right) \\
& =\prod_{m=l}^{n} \mathbb{P}_{p}\left(B^{m} \in W_{m}\right) \\
& \leq\left(p \varphi_{N, d}(p)\right)^{n-l+1} .
\end{aligned}
$$

Using Fatou's lemma and the fact that the collection of cubes in $V_{n}$ covers $\mathcal{C}^{c, \epsilon}$, we obtain (writing $\left|V_{n}\right|$ for the number of cubes in $V_{n}$ ):

$$
\begin{aligned}
\mathbb{E}_{p}\left(\mathcal{H}^{s}\left(\mathcal{C}^{c, \epsilon}\right)\right) & \leq \liminf _{n \rightarrow \infty} \mathbb{E}_{p}\left(\sum_{D_{\mathbf{k}}^{n} \in V_{n}} \operatorname{diam}\left(D_{\mathbf{k}}^{n}\right)^{s}\right) \\
& =\liminf _{n \rightarrow \infty}\left(\sqrt{d} N^{-n}\right)^{s} \mathbb{E}_{p}\left(\left|V_{n}\right|\right) \\
& \leq \liminf _{n \rightarrow \infty} d^{s / 2} N^{-s n}\left(p \varphi_{N, d}(p) N^{d}\right)^{n-l+1} \\
& =d^{s / 2} N^{-d(l-1)} p \varphi_{N, d}(p) \lim _{n \rightarrow \infty} N^{n\left(d+\frac{\log \left(p \varphi_{N, d}(p)\right)}{\log N}-s\right)} .
\end{aligned}
$$


The limit in (12) is finite if and only if

$$
s \geq d+\frac{\log \left(p \varphi_{N, d}(p)\right)}{\log N},
$$

showing that

$$
\operatorname{dim}_{\mathcal{H}}\left(\mathcal{C}^{c, \epsilon}\right) \leq d+\frac{\log \left(p \varphi_{N, d}(p)\right)}{\log N} \text { a.s. }
$$

It follows from (11) that

$$
\operatorname{dim}_{\mathcal{H}}\left(\mathcal{C}^{c}\right) \leq d+\frac{\log \left(p \varphi_{N, d}(p)\right)}{\log N} \quad \text { a.s. }
$$

and since $\varphi_{N, d}(p)<1$, the result follows from this and (3).

Proof of Theorem 1.2 If $\mathbb{P}_{p}\left(\mathcal{C}^{c}=\emptyset\right)=1$ then there is nothing to prove, so we assume that $\mathbb{P}_{p}\left(\mathcal{C}^{c} \neq \emptyset\right)>0$.

Let $Z_{n}$ be the number of retained cubes after $n$ steps of the fractal construction procedure and let $B_{1}, \ldots, B_{Z_{n}}$ denote the retained cubes. If the event $\left\{\operatorname{dim}_{\mathcal{H}}\left(\mathcal{C}^{c}\right) \geq \alpha\right\}$ occurs, then $\left\{\operatorname{dim}_{\mathcal{H}}\left(\mathcal{C}^{c} \cap B_{k}\right) \geq \alpha\right\}$ for at least one $k=1, \ldots, Z_{n}$ (see, e.g., [11]). Therefore,

$$
\mathbb{P}_{p}\left(\operatorname{dim}_{\mathcal{H}}\left(\mathcal{C}^{c}\right) \geq \alpha \mid Z_{n}=l\right)=1-\prod_{k=1}^{l}\left(1-\mathbb{P}_{p}\left(\operatorname{dim}_{\mathcal{H}}\left(\mathcal{C}^{c} \cap B_{k}\right) \geq \alpha \mid B_{k} \subset \mathcal{C}^{n}\right)\right.
$$

However, because of scale invariance, $\operatorname{dim}_{\mathcal{H}}\left(\mathcal{C}^{c} \cap B_{k}\right)$, conditioned on the event that $B_{k} \subset \mathcal{C}^{n}$, must have the same distribution as $\operatorname{dim}_{\mathcal{H}}\left(\mathcal{C}^{c}\right)$ and so in fact

$$
\mathbb{P}_{p}\left(\operatorname{dim}_{\mathcal{H}}\left(\mathcal{C}^{c}\right) \geq \alpha \mid Z_{n}=l\right)=1-\left(1-\mathbb{P}_{p}\left(\operatorname{dim}_{\mathcal{H}}\left(\mathcal{C}^{c}\right) \geq \alpha\right)\right)^{l}
$$

We can now write

$$
\begin{aligned}
\mathbb{P}_{p}\left(\operatorname{dim}_{\mathcal{H}}\left(\mathcal{C}^{c}\right) \geq \alpha\right) & =\sum_{l=1}^{N^{d n}} \mathbb{P}_{p}\left(\operatorname{dim}_{\mathcal{H}}\left(\mathcal{C}^{c}\right) \geq \alpha \mid Z_{n}=l\right) \mathbb{P}_{p}\left(Z_{n}=l\right) \\
& =\sum_{l=1}^{N^{d n}}\left(1-\left(1-\mathbb{P}_{p}\left(\operatorname{dim}_{\mathcal{H}}\left(\mathcal{C}^{c}\right) \geq \alpha\right)\right)^{l}\right) \mathbb{P}_{p}\left(Z_{n}=l\right) \\
& \geq\left[1-\left(1-\mathbb{P}_{p}\left(\operatorname{dim}_{\mathcal{H}}\left(\mathcal{C}^{c}\right) \geq \alpha\right)\right)^{n}\right] \mathbb{P}_{p}\left(Z_{n} \geq n\right) .
\end{aligned}
$$

This last quantity is bounded below by

$$
\left[1-\left(1-\mathbb{P}_{p}\left(\operatorname{dim}_{\mathcal{H}}\left(\mathcal{C}^{c}\right) \geq \alpha\right)\right)^{n}\right] \mathbb{P}_{p}\left(Z_{n} \geq n \mid \mathcal{C} \neq \emptyset\right) \mathbb{P}_{p}(\mathcal{C} \neq \emptyset) .
$$

As mentioned above, a.s. $Z_{n}^{1 / n} \rightarrow p N^{d}>1$ as $n \rightarrow \infty$ if $\mathcal{C} \neq \emptyset$. Therefore, if $\mathbb{P}_{p}\left(\operatorname{dim}_{\mathcal{H}}\left(\mathcal{C}^{c}\right) \geq \alpha\right)>0$, by taking the limit of (13) as $n \rightarrow \infty$, we conclude that 
$\mathbb{P}_{p}\left(\operatorname{dim}_{\mathcal{H}}\left(\mathcal{C}^{c}\right) \geq \alpha\right) \geq \mathbb{P}_{p}(\mathcal{C} \neq \emptyset)$. If $\alpha>0$, it follows that in fact $\mathbb{P}_{p}\left(\operatorname{dim}_{\mathcal{H}}\left(\mathcal{C}^{c}\right) \geq\right.$ $\alpha)=\mathbb{P}_{p}(\mathcal{C} \neq \emptyset)$. Letting $\phi(\alpha)=\mathbb{P}_{p}\left(\operatorname{dim}_{\mathcal{H}}\left(\mathcal{C}^{c}\right) \geq \alpha\right)$, we have that $\phi(0)=1$ and either $\phi(\alpha)=0$ or $\phi(\alpha)=\mathbb{P}_{p}(\mathcal{C} \neq \emptyset)$ when $\alpha>0$.

Now observe that $\mathcal{C}^{c} \neq \emptyset$ implies $\operatorname{dim}_{\mathcal{H}}\left(\mathcal{C}^{c}\right) \geq 1$ (see, e.g., Proposition 4.1 of [11]). Since the inverse implication is obvious, we conclude that $\phi(1)=\mathbb{P}_{p}\left(\mathcal{C}^{c} \neq \emptyset\right)>0$ (where the last inequality follows from the assumption made at the beginning of the proof) and that $\mathbb{P}_{p}\left(\mathcal{C}^{c} \neq \emptyset\right)=\mathbb{P}_{p}(\mathcal{C} \neq \emptyset)$. Hence, since $\left\{\mathcal{C}^{c} \neq \emptyset\right\} \subset\{\mathcal{C} \neq \emptyset\}$, we obtain that

$$
\left\{\mathcal{C}^{c} \neq \varnothing\right\}=\{\mathcal{C} \neq \varnothing\}
$$

up to a set of probability 0 .

Moreover, since $\phi(\alpha)=\mathbb{P}_{p}(\mathcal{C} \neq \emptyset)$ for at least $\alpha \leq 1$, and $\left\{\operatorname{dim}_{\mathcal{H}}\left(\mathcal{C}^{c}\right) \geq \alpha\right\} \subset$ $\{\mathcal{C} \neq \emptyset\}$ for $\alpha>0$, we also obtain that $\left\{\operatorname{dim}_{\mathcal{H}}\left(\mathcal{C}^{c}\right) \geq \alpha\right\}=\{\mathcal{C} \neq \emptyset\}$, up to a set of probability 0 , for every $\alpha>0$ such that $\mathbb{P}_{p}\left(\operatorname{dim}_{\mathcal{H}}\left(\mathcal{C}^{c}\right) \geq \alpha\right)>0$.

Writing

$$
\beta:=\sup \left\{\alpha: \mathbb{P}_{p}\left(\operatorname{dim}_{\mathcal{H}}\left(\mathcal{C}^{c}\right) \geq \alpha\right)>0\right\},
$$

we conclude from the above observations that, up to a set of probability 0 , if $\mathcal{C}^{c} \neq \emptyset$ then $\operatorname{dim}_{\mathcal{H}}\left(\mathcal{C}^{c}\right)=\beta$, with $\beta \geq 1$ as a consequence of the assumption that $\mathbb{P}_{p}\left(\operatorname{dim}_{\mathcal{H}}\left(\mathcal{C}^{c}\right) \neq \emptyset\right)>0$ and the fact that $\operatorname{dim}_{\mathcal{H}}\left(\mathcal{C}^{c}\right) \geq 1$ as soon as $\mathcal{C}^{c} \neq \emptyset$.

Theorem 1.2 and the proof of Theorem 1.1 have an interesting corollary which links $\operatorname{dim}_{\mathcal{H}}\left(\mathcal{C}^{c}\right)$ to the discontinuity at the critical point $p_{c}(N, d)$. (This is the corollary that was announced in the introduction.)

Corollary 2.1 Let $\Delta$ denote the a.s. Hausdorff dimension of $\mathcal{C}^{c}$ when $p=p_{c}(N, d)$ and $\mathcal{C}^{c} \neq \emptyset$. Then,

$$
\varphi_{N, d}\left(p_{c}(N, d)\right) \geq \frac{1}{N^{d-\Delta}}
$$

Proof Let $p_{b}(N, d):=\inf \left\{p \leq 1: \varphi_{N, d}(p)>0\right\}$. Theorem 4.1 of [5] and the observation preceding it show that $p_{b}(N, d)=p_{c}(N, d)$. Moreover, it follows from [5] that $\varphi_{N, d}\left(p_{c}(N, d)\right)>0$. Combining these observations with the last line of the proof of Theorem 1.1, we obtain that, for $p \geq p_{c}(N, d)$,

$$
\varphi_{N, d}(p) \geq p \varphi_{N, d}(p) \geq \frac{1}{N^{d-\Delta}} .
$$

Remark For Theorem 1.1 we use the result from [5] that for $p=p_{c}(N, d), \mathbb{P}_{p}\left(\mathcal{C}^{c} \neq\right.$ $\emptyset)>0$. It is possible to prove the result without this prior knowledge, as follows. We can start with the observation from [5] that $p_{c}=p_{b}$. We can then prove Theorem 1.1 in the case of $p>p_{c}$ and from the last line of that proof we get that $\varphi_{N, d}(p) \geq \frac{1}{N^{d-1}}$, using Corollary 2.1 and the fact that the Hausdorff-dimension of a connected set consisting of more than one point is at least 1 (see, e.g., Proposition 4.1 of [11]). Using this uniform bound and a right-continuity argument similar to the ones in [5], we conclude that in fact $\varphi_{N, d}\left(p_{c}\right) \geq \frac{1}{N^{d-1}}$. Hence we can conclude that for $p=$ 
$p_{c}(N, d), P_{p}\left(\mathcal{C}^{c} \neq \emptyset\right)>0$, and go through the proof once more to obtain the same result as above.

Proof of Proposition 1.3 We again use the fact that $\mathcal{C}^{c, \epsilon}$ is contained in the union of the cubes in $V_{n}$, defined in the proof of Theorem 1.1, and therefore, $M_{N^{-n}}=$ $M_{N^{-n}}\left(\mathcal{C}^{c, \epsilon}\right) \leq\left|V_{n}\right|$. First observe that

$$
\liminf _{\delta \rightarrow 0} \frac{\log M_{\delta}}{-\log \delta}=\liminf _{n \rightarrow \infty} \frac{\log M_{N^{-n}}}{-\log N^{-n}} .
$$

By Fatou's lemma and Jensen's inequality we get along the same lines as in the last part of the proof of Theorem 1.1 (using the same $l$ as in that proof) that

$$
\begin{aligned}
\mathbb{E}_{p}\left(\liminf _{n \rightarrow \infty} \frac{\log M_{N^{-n}}}{-\log N^{-n}}\right) \\
\leq \liminf _{n \rightarrow \infty} \mathbb{E}_{p}\left(\frac{\log M_{N^{-n}}}{-\log N^{-n}}\right) \leq \liminf _{n \rightarrow \infty} \frac{\log \mathbb{E}_{p}\left(M_{N^{-n}}\right)}{-\log N^{-n}} \\
\leq \liminf _{n \rightarrow \infty} \frac{\log \mathbb{E}_{p}\left(\left|V_{n}\right|\right)}{n \log N} \leq \liminf _{n \rightarrow \infty} \frac{\log \left(p \varphi_{N, d}(p) N^{d}\right)^{n-l+1}}{n \log N} \\
=\frac{\log \left(p \varphi_{N, d}(p) N^{d}\right)}{\log N}=d+\frac{\log \left(p \varphi_{N}(p)\right)}{\log N} .
\end{aligned}
$$

Since $\varphi_{N, d}(p)<1$ it follows that $d+\log \left(p \varphi_{N, d}(p)\right) / \log N<d+\log p / \log N$.

\section{Proof of Theorems 1.4 and 1.5}

In this section and the next one we work in two dimensions, that is, $d=2$. We will be using the machinery in [1] concerning scaling limits of systems of random curves.

One part of the argument is to apply some of the machinery developed in [1] in order to show that the sequence of measures $\mu_{n}$ defined in the introduction has subsequential weak limits, and we first deal with this issue. After that, we combine the existence of weak limits with the a.s. limit behavior of the fractal process in order to draw the final conclusions.

Let $B(x, r)$ denote a closed square centred at $x$ with side length $r$ and $B^{\circ}(x ; r)$ its interior. For $R>r$, let $A(x ; r, R):=B(x, R) \backslash B^{\circ}(x, r)$ be an annulus. The basic estimate is the following, from which everything else will follow.

Lemma 3.1 Let $p \geq p_{c}(N, 2)$. There exists a sequence $\lambda(1), \lambda(2), \ldots$ with $\lim _{k \rightarrow \infty} \lambda(k)=\infty$ and finite constants $K_{k}$ such that the following bound holds uniformly for all $r \leq R \leq 1$ with $r$ small enough, and all $x$ :

$$
\limsup _{n \rightarrow \infty} \mathbb{P}_{p}\left(\mathcal{F}_{n} \text { contains } k \text { disjoint crossings of } A(x ; r, R)\right) \leq K_{k}\left(\frac{r}{R}\right)^{\lambda(k)} .
$$


Fig. 3 The annulus $A_{n_{1}}$ with 3 level- $n_{1}$ components, drawn in dark grey

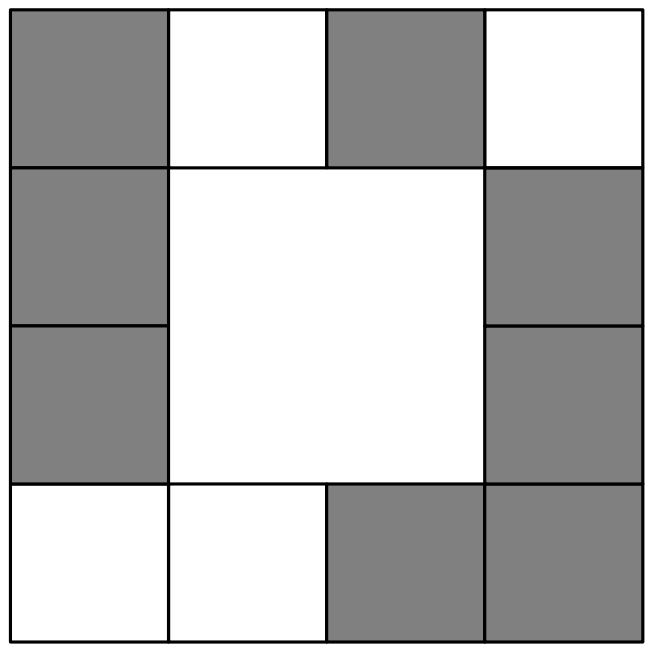

Proof We are looking for a collection of mutually disjoint annuli (all contained in $B(x, R)$ and "surrounding" $B(x, r))$ of the form $A\left(y ; 2 N^{-n}, 4 N^{-n}\right)$, where $y$ is a corner point of some square $D_{\mathbf{k}}^{n}$ of the fractal construction. It is not hard to see that for any $x \in[0,1]^{2}$, we can find such a collection with at least $M:=c \log (R / r)$ elements, for a suitable positive constant $c$. For small enough $r, M$ is at least 1 . For a given $x$, we denote these annuli by $A_{n_{1}}, A_{n_{2}}, \ldots, A_{n_{M}}$, where the indices $n_{1}<n_{2}<\cdots<n_{M}$ refer to the $n$ associated with the annuli. The idea is that if there are $k$ disjoint crossings of $A(x ; r, R)$, then each annulus $A_{n_{i}}$ must also be crossed by $k$ disjoint crossings in $\mathcal{F}_{n}$. This is exponentially unlikely in the number of annuli, as we will show now.

We first consider the annulus $A_{n_{1}}$. Let $n>n_{1}$, and perform the fractal process until level $n_{1}$ (inclusive). The annulus $A_{n_{1}}$ consists of 12 level- $n_{1}$ squares (Fig. 3), some of which are in $\mathcal{C}^{n_{1}}$ and some of which are not. Now observe that the collection of black (retained) squares of level $n_{1}$ in $A_{n_{1}}$ is partitioned into at most 6 "level$n_{1}$ components", where two neighboring retained level- $n_{1}$ squares are in the same component if they share an edge: see Fig. 3 for an illustration of the event that the annulus contains 3 such level- $n_{1}$ components.

Now let $k$ be large enough (exactly how large will become clear soon). If $A_{n_{1}}$ is, after $n$ iterations, crossed by $k$ disjoint interface segments, then at least one of the level- $n_{1}$ components must be crossed by at least $k / 6$ such segments, since there are at most 6 components to accommodate these crossings (we should write $\lfloor k / 6\rfloor$ but we ignore these details for the sake of notational convenience). Between the interface crossings, one has alternating black (retained) and white (discarded) crossings of the annulus. This implies that the component with at least $k / 6$ interface crossings is, after $n$ iterations, also crossed by at least $k / 12-1$ white crossings which are "between" the "first" and "last" of the $k / 6$ interface crossings of said component.

The point of considering the level- $n_{1}$ components introduced above is that they are disjoint and separated by vacant squares, so that the fractal constructions inside them from level $n_{1}$ on are independent of each other, and none of the interface segments crossing $A_{n_{1}}$ can intersect more than one of them. Therefore, adding extra retained 
level- $n_{1}$ squares in $A_{n_{1}}$ does not affect the $k / 12-1$ white crossings in the component with at least $k / 6$ interface crossings. This implies that the probability of having at least $k$ interface crossings in $A_{n_{1}}$ after $n$ iterations is bounded above by the probability of having at least $k / 12-1$ white crossings after $n$ iterations, conditioned on having full retention in $A_{n_{1}}$ up to level $n_{1}$.

However, scaling tells us that, if we condition on retention until level $n_{1}$, the probability in question is the same as the probability of having at least $k / 12-1$ disjoint white components crossing $\bar{A}:=A((0,0) ; 2,4)$ when we perform $n-n_{1}$ iterations of the fractal process in $[-2,2]^{2}$ rather than in $[0,1]^{2}$, seen as the union of 16 independent fractal processes on the 16 unit squares making up $[-2,2]^{2}$. For these white crossing components we can use the BK inequality (see [13] - a similar BK inequality for black crossing is not available) and deduce that the probability of having $k / 12-1$ of such white crossing components is bounded above by the probability of having at least one, raised to the power $k / 12-1$. This then finally leads to the estimate that

$$
\mathbb{P}_{p}\left(\mathcal{F}_{n} \text { contains } k \text { disjoint crossings of } A(x ; r, R)\right)
$$

is bounded above by

$$
\mathbb{P}_{p}\left(\bar{A} \text { is crossed by a white component after } n-n_{1} \text { steps }\right)^{k / 12-1} \text {. }
$$

If there is a white component crossing $\bar{A}$, then there is no black circuit surrounding the origin in $\bar{A}$. The probability of having such a black circuit after $n-n_{1}$ iterations is at least as large as the probability to have such a circuit in the limit, and by the weak RSW theorem for fractal percolation in [10] and the FKG inequality, we have that for $p \geq p_{c}(N, 2)$ this probability is strictly positive. It follows that there exists $\alpha<1$ such that (16) is bounded above by $\alpha^{k / 12-2}$, uniformly in $n>n_{1}$.

Next we consider $A_{n_{1}}$ and $A_{n_{2}}$ simultaneously. Take $n>n_{2}$. The probability to have $k$ interface crossings in $A_{n_{1}}$ and also in $A_{n_{2}}$ is the probability that this happens in $A_{n_{1}}$ multiplied by the probability that this happens in $A_{n_{2}}$ conditioned on the fact that it happens in $A_{n_{1}}$. We can treat this conditional probability exactly as above: we can change the conditioning into one involving complete retention inside $A_{n_{2}}$ until level $n_{2}$, to get an upper bound. It follows that the probability that both $A_{n_{1}}$ and $A_{n_{2}}$ have $k$ interface crossings is bounded above by the square of the individual bounds, that is, by $\left(\alpha^{k / 12-1}\right)^{2}$.

We continue in the obvious way now, leading to the conclusion that for $n>n_{M}$, the probability that all annuli $A_{n_{1}}, \ldots, A_{n_{M}}$ are crossed by $k$ interface crossings, is bounded above by

$$
\left(\alpha^{k / 12-1}\right)^{c \log (R / r)} .
$$

A little algebra shows that this is equal to

$$
\left(\frac{r}{R}\right)^{c \log \left(\alpha^{1-k / 12}\right)}
$$

and this is a bound of the required form, with $\lambda(k)=c \log \left(\alpha^{1-k / 12}\right)$. 
We now describe how the existence of subsequential weak limits of the sequence $\mu_{n}$ follows from this lemma. (This is well known but perhaps not immediately obvious from the literature, hence our summary for the convenience of the reader.) For $\epsilon>0$ and positive integers $k, n$ define

$$
r_{\epsilon, k}^{n}:=\inf \left(\left\{0<r \leq 1: \begin{array}{l}
\text { some annulus } A\left(x ; r^{1+\epsilon}, r\right), x \in[0,1]^{2}, \text { is } \\
\text { crossed by } k \text { disjoint crossings in } \mathcal{F}_{n}
\end{array}\right\}, 1\right) .
$$

It follows exactly as in [1] or [14] that, as a consequence of Lemma 3.1, for any $\epsilon>0$, for large enough $k$ the random variables $r_{\epsilon, k}^{n}$ are stochastically bounded away from zero as $n \rightarrow \infty$, that is

$$
\lim _{u \rightarrow 0} \limsup _{n \rightarrow \infty} \mathbb{P}_{p}\left(r_{\epsilon, k}^{n} \leq u\right)=0
$$

Note that in Lemma 3.1 we have the result (15) only for $r$ small enough, while the corresponding hypothesis $\mathrm{H} 1$ in [1] is stated without that restriction. Our Lemma 3.1 however is sufficient to prove (17), since the latter concerns the behavior of $\mathbb{P}_{p}\left(r_{\epsilon, k}^{n} \leq\right.$ $u)$ as $u \rightarrow 0$.

Note also that Hypothesis H1 is used in (3.4) in [1] only for annuli whose inner radius equals $3 r_{n}^{1+\epsilon}$, with $r_{n}=2^{-n} \leq u$ for $u>0$. By taking $u$ sufficiently small in the proof of Lemma 3.1 in [1], also the inner radius $3 r_{n}^{1+\epsilon}$ becomes sufficiently small and we can apply our Lemma 3.1. Hence, (17) follows from our Lemma 3.1 by the same arguments used in [1] or [14].

As is shown in [1] (see in particular their proof of Theorem 1.1 and Eq. (1.7) in their Remark (ii) following the statement of Theorem 1.1, but note the typo in Eq. (1.7), where $d / \lambda(1)$ should be $\lambda(1) / d$ ), Eq. (17) implies the following result.

Theorem 3.2 [1] For any $\epsilon>0$, all curves $\Gamma \in \mathcal{F}_{n}$ can be parameterized by continuous functions $\gamma:[0,1] \rightarrow[0,1]^{2}$ such that for each curve, for all $0 \leq t_{1} \leq t_{2} \leq 1$,

$$
\left|\gamma\left(t_{1}\right)-\gamma\left(t_{2}\right)\right| \leq k_{\epsilon}^{n}\left|t_{1}-t_{2}\right|^{\frac{1}{2+\epsilon}}
$$

where the random variables $k_{\epsilon}^{n}$ are stochastically bounded as $n \rightarrow \infty$, that is,

$$
\lim _{u \rightarrow \infty} \limsup _{n \rightarrow \infty} \mathbb{P}_{p}\left(k_{\epsilon}^{n} \geq u\right)=0 .
$$

Once we have this result, we use Theorem 5.7 in [14] and Theorem 1.1 in [1] to conclude that the sequence of measures $\left\{\mu_{n}\right\}_{n \geq 1}$ is tight. Since $\Sigma$ is separable, it then follows from Prohorov's theorem that for every sequence $n_{k} \rightarrow \infty$ there exists a subsequence $n_{k_{l}} \rightarrow \infty$ such that $\mu_{n_{k_{l}}}$ converges weakly to a probability measure on $\Sigma$.

Finally, from the fact that the $k_{\epsilon}^{n}$ are stochastically bounded and the fact that the collection of curves with a given Hölder exponent is compact, we have (see also [14] and [1]) that if we sample from any such weak limit, all curves $\gamma$ in the sample can be parameterized in such a way that

$$
\left|\gamma\left(t_{1}\right)-\gamma\left(t_{2}\right)\right| \leq M\left|t_{1}-t_{2}\right|^{\alpha}
$$


where $M$ is a random number common to all curves in the same sample, and $\alpha$ is a (non-random) constant.

Next we combine the above weak convergence with the a.s. convergence of the retained squares (as compact sets) in the fractal process. Let $(S, H)$ denote the metric space of compact subsets of $[0,1]^{d}$ with the Hausdorff distance $H$ and let ( $\Sigma$, Dist) be as defined after (9). Furthermore, let the function $g: \Sigma \mapsto S$ be defined by $g(\mathcal{F})=$ $\bigcup_{\gamma \in \mathcal{F}} \operatorname{Image}(\gamma)$ and define $F_{n}=g\left(\mathcal{F}_{n}\right)$.

Our next result concerns weak convergence of $\left(\mathcal{F}_{n}, F_{n}\right)$ where we use the product topology on $\Sigma \times S$.

Lemma 3.3 The distribution of $\left(\mathcal{F}_{n}, F_{n}\right)$ converges weakly along a subsequence. Furthermore, any pair $(\mathcal{F}, F)$ of random variables sampled from any such weak limit a.s. satisfies $F=g(\mathcal{F})$.

Proof We already know that the distribution $\mu_{n}$ of $\mathcal{F}_{n}$ converges weakly along a subsequence. Using (8) and (9), if $\operatorname{Dist}\left(\mathcal{F}, \mathcal{F}^{\prime}\right) \leq \delta$, this immediately implies that $H\left(F, F^{\prime}\right) \leq \delta$ since the images of any two curves $\gamma_{1}, \gamma_{2}$ such that $D\left(\gamma_{1}, \gamma_{2}\right) \leq \delta$ are within Hausdorff distance $\delta$. This proves that $g$ is continuous.

The convergence in distribution of $\mathcal{F}_{n}$ along some subsequence $n_{k}$ to a limit $\mathcal{F}$ implies the existence of coupled versions $X_{k}$ and $X$ of $\mathcal{F}_{n_{k}}$ and $\mathcal{F}$ respectively, such that $X_{k}$ converges to $X$ in probability as $k \rightarrow \infty$ (see, e.g., Corollary 1 in [3]). Moreover, since $g$ is continuous, $g\left(X_{k}\right) \stackrel{\text { dist. }}{=} F_{n_{k}}$ converges in probability to $g(X) \stackrel{\text { dist. }}{=} g(\mathcal{F})$ as $k \rightarrow \infty$. This implies convergence in probability of the vector $\left(X_{k}, g\left(X_{k}\right)\right)$ to $(X, g(X))$, which yields the joint convergence in distribution of $\left(\mathcal{F}_{n_{k}}, F_{n_{k}}\right)$ to some $\operatorname{limit}(\mathcal{F}, F)$ with $F=g(\mathcal{F})$ a.s.

Proof of Theorem 1.4 According to Lemma 3.3 there exists a subsequence $\left\{n_{k}\right\}_{k \geq 1}$ such that $\left(\mathcal{F}_{n_{k}}, F_{n_{k}}\right)$ converges weakly to some limit $(\mathcal{F}, F)$ where $F$ is a.s. the union of the images of $\mathcal{F}$. Furthermore, we claim that a.s.

$$
H\left(F_{n}, \mathcal{C}\right) \rightarrow 0 \quad \text { as } n \rightarrow \infty
$$

where $F_{n}=g\left(\mathcal{F}_{n}\right)$. To see this, let $F_{n}^{\epsilon}$ denote the $\epsilon$-neighborhood of $F_{n}$ and note that $\mathcal{C} \not \subset F_{n}^{\epsilon}$ implies that there exists an $x \in \mathcal{C}$ such that $B(x, \epsilon) \cap F_{n}=\emptyset$ and so $B(x, \epsilon) \subset \mathcal{C}^{n}$; otherwise an interface curve would be closer to $x$ than $\epsilon$. It is however easy to prove that the probability that there exists a ball of radius $\epsilon$ in $\mathcal{C}^{n}$ goes to 0 . For the other direction, let $D=D(\mathcal{C}, \epsilon)$ be the complement of the open $\epsilon$-neighborhood of $\mathcal{C}$. Obviously $D \cap \mathcal{C}^{n}$ is a compact set (if non-empty) and furthermore $D \cap \mathcal{C}^{n+1} \subset D \cap \mathcal{C}^{n}$ for every $n$. Therefore, by compactness, if $D \cap \mathcal{C}^{n} \neq \emptyset$ for every $n$ then $\bigcap_{n=1}^{\infty} D \cap \mathcal{C}^{n} \neq \emptyset$ and so there are points in $\mathcal{C}$ that are also in $D$, which is a contradiction. Therefore, the open $\epsilon$-neighborhood of $\mathcal{C}$ will eventually contain $\mathcal{C}^{n}$ and hence also $F_{n}$.

Since $F_{n_{k}}$ converges weakly to $F$ and a.s. to $\mathcal{C}$ (because of (19)), we conclude that $F$ and $\mathcal{C}$ have the same distribution, and hence $\mathcal{C}$ has the same distribution as $g(\mathcal{F})$. The fact that (as noted above), a.s., a realization from $\mathcal{F}$ only contains Hölder continuous curves satisfying (18) finishes the proof. 
Proof of Theorem 1.5. Let $v_{n}$ be the distribution of $\sigma_{n}$. We repeat the proof of Theorem 1.4, with the distance (9) replaced by (8). This shows that conditioned on the existence of a left-right crossing for all $n, v_{n}$ has subsequential weak limits as $n \rightarrow \infty$ and in addition that any such limit assigns probability 1 to Hölder continuous curves.

\section{Proof of Theorem 1.6}

We will start by showing that the fractal percolation process satisfies Hypothesis $\mathrm{H} 2$ in [1], from which Theorem 1.6 follows. The hypothesis concerns probabilistic bounds on crossings in the long direction of certain rectangles.

A collection of sets $\left\{A_{i}\right\}$ is called well-separated if for all $i$ the distance of each set $A_{i}$ to the other sets $\left\{A_{j}\right\}_{j \neq i}$ is at least as large as the diameter of $A_{i}$. Hypothesis $\mathrm{H} 2$ in [1] reads as follows.

Hypothesis 4.1 There exist $\sigma>0$ and some $\rho<1$ such that for every collection of $k$ well-separated rectangles $A_{1}, \ldots, A_{k}$ of width $l_{1}, \ldots, l_{k}$ and length $\sigma l_{1}, \ldots, \sigma l_{k}$, the following inequality holds:

$$
\limsup _{n \rightarrow \infty} \mathbb{P}_{p}\left(\mathcal{F}_{n} \text { contains a long crossing in each of } A_{1}, \ldots, A_{k}\right) \leq \rho^{k} .
$$

\section{Lemma 4.2 Hypothesis 4.1 holds for interface curves in the fractal process.}

Proof We assume without loss of generality that $l_{1} \geq l_{2} \geq \cdots \geq l_{k}$. Let $n_{i}$ be the smallest integer $n$ for which all rectangles of dimensions $l_{i}$ and $\sigma l_{i}$ contain an $n$-level square of the fractal construction, and define $\mathcal{A}_{i}$ as the event of complete retention until iteration step $n_{i}$. Let $C R_{n}^{I}\left(A_{i}\right)$ denote the event that the closed rectangle $A_{i}$ is crossed in the long direction by an interface segment after $n$ iterations of the fractal process, and define $C R_{n}^{B}\left(A_{i}\right)$ similarly for a black crossing.

Note that by the fact that $A_{1}, \ldots, A_{k}$ are well-separated and by the choice of $n_{1}$, given $\mathcal{A}_{1}$, the event $C R_{n}^{B}\left(A_{1}\right)$ is, when $n>n_{1}$, conditionally independent of the events $C R_{n}^{B}\left(A_{2}\right), \ldots, C R_{n}^{B}\left(A_{k}\right)$, since no level- $n$ square intersects more than one rectangle. Note also that, if a rectangle is crossed in the long direction by an interface segment, then it also contains a black crossing in the long direction. Using these two facts and the FKG inequality gives, for $n>n_{1}$,

$$
\begin{aligned}
\mathbb{P}_{p}\left(\bigcap_{i=1}^{k} C R_{n}^{I}\left(A_{i}\right)\right) & \leq \mathbb{P}_{p}\left(\bigcap_{i=1}^{k} C R_{n}^{B}\left(A_{i}\right)\right) \\
& \leq \mathbb{P}_{p}\left(\bigcap_{i=1}^{k} C R_{n}^{B}\left(A_{i}\right) \mid \mathcal{A}_{1}\right) \\
& =\mathbb{P}_{p}\left(C R_{n}^{B}\left(A_{1}\right) \mid \mathcal{A}_{1}\right) \mathbb{P}_{p}\left(\bigcap_{i=2}^{k} C R_{n}^{B}\left(A_{i}\right) \mid \mathcal{A}_{1}\right) .
\end{aligned}
$$


Since $l_{1} \geq l_{2} \geq \cdots \geq l_{k}$, we have $n_{1} \leq n_{2} \leq \cdots \leq n_{k}$ and hence $\mathcal{A}_{1} \supset \mathcal{A}_{1} \supset \cdots \supset \mathcal{A}_{k}$. It follows as before that for $n>n_{2}$ we have

$$
\begin{aligned}
\mathbb{P}_{p}\left(\bigcap_{i=2}^{k} C R_{n}^{B}\left(A_{i}\right) \mid \mathcal{A}_{1}\right) & \leq \mathbb{P}_{p}\left(\bigcap_{i=2}^{k} C R_{n}^{B}\left(A_{i}\right) \mid \mathcal{A}_{2}\right) \\
& =\mathbb{P}_{p}\left(C R_{n}^{B}\left(A_{2}\right) \mid \mathcal{A}_{2}\right) \mathbb{P}_{p}\left(\bigcap_{i=3}^{k} C R_{n}^{B}\left(A_{i}\right) \mid \mathcal{A}_{2}\right) .
\end{aligned}
$$

We repeat this procedure $k-2$ more times, finally obtaining, for $n>n_{k}$, that

$$
\mathbb{P}_{p}\left(\bigcap_{i=1}^{k} C R_{n}^{I}\left(A_{i}\right)\right) \leq \prod_{i=1}^{k} \mathbb{P}_{p}\left(C R_{n}^{B}\left(A_{i}\right) \mid \mathcal{A}_{i}\right)
$$

It remains to show that $\mathbb{P}_{p}\left(C R_{n}^{B}\left(A_{i}\right) \mid \mathcal{A}_{i}\right)$ is uniformly bounded above by some $\rho<1$. This can be seen as follows. Let, for each $i, W_{i}$ be a smallest collection of level- $\left(n_{i}+1\right)$ squares in the fractal process with the property that if each of the squares in $W_{i}$ is white, then $A_{i}$ cannot be crossed by a black path in the long direction. It is easy to see from the choice of $n_{i}$ that the cardinality of $W_{i}$ is uniformly bounded above and hence that the probability that all squares in $W_{i}$ are white is uniformly bounded below by some positive number. It follows that the probability of a long black crossing in $A_{i}$ is uniformly bounded above by some number strictly smaller than 1 .

Proof of Theorem 1.6 The result follows from Lemma 4.2 and Theorem 1.3 in [1].

Acknowledgements $\quad$ F. Camia's research was supported by NWO VIDI Grant 639.032.916.

M. Joosten's research was supported by NWO Grant 613.000.601.

F. Camia thanks L. Chayes for suggesting the argument that led to the proof of Eq. (6).

Open Access This article is distributed under the terms of the Creative Commons Attribution License which permits any use, distribution, and reproduction in any medium, provided the original author(s) and the source are credited.

\section{References}

1. Aizenman, M., Burchard, A.: Hölder regularity and dimension bounds for random curves. Duke Math. J. 99, 419-453 (1999)

2. Athreya, K.B., Ney, P.E.: Branching Processes. Springer, Berlin (1972)

3. Billingsley, P.: Weak Convergence of Measures: Applications in Probability. Society for Industrial and Applied Mathematics, Philadelphia (1971)

4. Broman, E.I., Camia, F.: Large- $N$ limit of crossing probabilities, discontinuity, and asymptotic behavior of threshold values in Mandelbrot's fractal percolation process. Electr. J. Prob. 13, 980-999 (2008)

5. Broman, E.I., Camia, F.: Universal behavior of connectivity properties in fractal percolation models. Electr. J. Prob. 15, 1396-1414 (2010)

6. Camia, F., Newman, C.M.: Two-dimensional critical percolation: the full scaling limit. Commun. Math. Phys. 268, 1-38 (2006) 
7. Chayes, L.: On the length of the shortest crossing in the super-critical phase of Mandelbrot's percolation process. Stoch. Proc. Rel. Fields 61, 25-43 (1994)

8. Chayes, J.T., Chayes, L., Durrett, R.: Connectivity properties of Mandelbrot's percolation process. Prob. Th. Rel. Fields 77, 307-324 (1988)

9. Dekking, F.D., Grimmett, G.R.: Superbranching processes and projections. Prob. Th. Rel. Fields 78, 335-355 (1988)

10. Dekking, F.D., Meester, R.W.J.: On the structure of Mandelbrot's percolation process and other random Cantor sets. J. Stat. Phys. 58, 1109-1126 (1990)

11. Falconer, K.J.: Fractal Geometry. Wiley, Chichester (1990)

12. Mandelbrot, B.B.: The Fractal Geometry of Nature. Freeman, San Francisco (1983)

13. Meester, R.W.J.: Connectivity in fractal percolation. J. Theor. Prob. 5, 775-789 (1992)

14. Sun, N.: Conformally invariant scaling limits in planar critical percolation. Probab. Surv. 8, 155-209 (2011). arXiv:0911.0063v1 (2009) 